\title{
Collaborative Design and Manufacture Supported by Multiple Web/Internet Techniques
}

\author{
Daizhong Su, Jiansheng Li,Yu Xiong and Yongjun Zheng \\ Advanced Design and Manufacturing Engineering Centre, SBE \\ Nottingham Trent University, UK \\ URL: http://www.admec.ntu.ac.uk E-mail: daizhong.su@ntu.ac.uk
}

\begin{abstract}
A research project in Web-enable collaborative design and manufacture has been conducted. The major tasks of the project include development of a Web-enable environment for collaboration, online collaborative CAD/CAM, remote execution of large size programs (RELSP), and distributed product design. The tasks and Web/Internet techniques involved are presented first, followed by detail description of two approaches developed for implementation of the research: (1) a client-server approach for RELSP, where the following Internet techniques are utilized: CORBA, Microsoft's Internet information server, Tomcat server, JDBC and ODBC; (2) Web-Services supported collaborative $\mathrm{CAD}$ which enables geographically dispersed designers jointly conduct a design task in the way of speaking and seeing each other and instantaneously modifying the $\mathrm{CAD}$ drawing online.
\end{abstract}

\section{Introduction}

Collaborative design and manufacture supported by the Web/Internet technologies has been attracting researchers' and industrialists' great attention. For example, Chan, Zhang and $\mathrm{Li}$ proposed an agent and CORBA based integration platform for agile manufacture [1], Zaremba and Morel investigated the integration and control of intelligence in distributed manufacturing [2], Hou, Su and Hull integrated Web-based techniques and business strategies into the development of a network supported system for supplier selection [3], and so on. Those efforts revealed the increasing demands for Web/Internet technologies and the latest development in this area.

The authors currently involved in two EU-China collaboration projects financially supported by the European Commission's Asia IT\&C [4] and Asia-Link [5] programmes. Research in Web-enable collaboration is a major task of the two projects. The aim of the research is to improve co-operation between China and Europe by improving Information Science Interconnectivity in the area of intelligent manufacturing. The main activities include: (1) Development of a Web-enabled environment for geographically dispersed teams to collaborate over the Internet, (2) Research and development into enabling ICT for collaborative design and manufacturing and their implementation with the Web-enabled environment, and (3) Case studies to apply the methods, procedures and ICT tools developed.

In the following sections, the major tasks of the research and related Web/Internet techniques are presented first, followed by detail description of the application of two 
techniques related to a client-server approach and Web-service for collaborative design and manufacture.

\section{Overview of the Web/Internet Techniques Involved}

This research includes the following major tasks where the Web and Internet techniques are heavily involved:

(a) Web-enabled Environment (WEE) for Collaborative Design and Manufacture. In the development of the WEE, it has been considered that the partners are not only dispersed geographically but may also work with different platforms, operating systems, protocols and languages. As a large heterogeneous platform for collaboration and integration over the Internet, the WEE has the following features: scalability, openness, heterogeneity, resources accessibility and inter-operation, legacy codes reusability and artificial intelligence [6].

(b) Online collaborative computer aided design. Current development in this area includes two aspects: (1) Internet-driven collaborative design with 3D feature modeling including form feature, parameterized form feature and parameter list of the form features [7], and (2) Web-service supported online collaborative computer aided design which is further detailed in Section 4 below.

(c) Web-enable collaborative computer aided manufacture. This includes prediction and simulation of manufacturing processes and production planning both during the conceptual design when design data are incomplete and during the later stages when the design has matured after several design iterations. The Web-based computer aided process planning and a remote monitoring system are both considered. The approach developed is illustrated with a case study of manufacturing mechanical components using Parallel Kinematics Machine [8].

(d) Effective remote-execution of large size programs. In order to achieve best product design and lowest production costs, some large-sized ICT tools and programs, such as design optimisation and finite element analysis software, are often used in the design phase of product development. They are time-consuming in computation and may not be valid to download due to some reasons such as copyright, large size of the software and the limited network bandwidth. Two approaches have been developed to remotely execute such software in an effective way: (1) a CGI (common gateway interface) approach [9], and (2) a client-server approach where the Internet techniques involved include CORBA, Mirosoft's Internet Information Server, Tomcat (a Servletenabled server), JDBC (JAVA database connectivity) and ODBC (open database connectivity), which is presented in Section 3 below.

(e) Web-enabled distributed product design. This includes dynamic databases, product data management (PDM) and knowledge based engineering (KBE). Within the system, users interact with the server through the Middleware. This server includes a JAVA application which interacts with the dynamic database and the PDM system. The dynamic databases contain all the necessary knowledge for product design such as design rules and process parameters. The basic PDM features are implemented in a program that resides in the Server. This program is therefore the connection between CAD and CAM users, as well as the KBE and dynamic database 
for which it gives writing permissions, etc. The KBE modules are for specific part families and production processes. Current progress in this area is reported in [10].

\section{A Client-Sever Approach for Remote Execution of a Large Size Program}

\subsection{Structure of the system}

In engineering practice, there are numbers of large computing programs used in variety applications such as optimisation, analysis, design, drafting, etc. Most of them are stand-alone packages, written in conventional programming languages such as $\mathrm{C}++$ or Fortran, and thus are not Web-enabled. Such an application normally consists of three parts:

- a graphical user interface (GUI);

- a main computing program; and

- data files or databases.

In order to make the applications executable over the Internet, the following are required:

- The user can easily access the application through an Internet explorer, regardless the platform type of the user machine.

- The user can easily input data, monitor the executing process, and receive resultant data in a demanding way.

- Without impaction on program execution in case of the interruption of Internet.

- Multiple users' complementation and authentication.

To meet the above requirements, a client/server system has been developed in Java and HTTP by the authors. The structure of the system is shown in Figure 1.

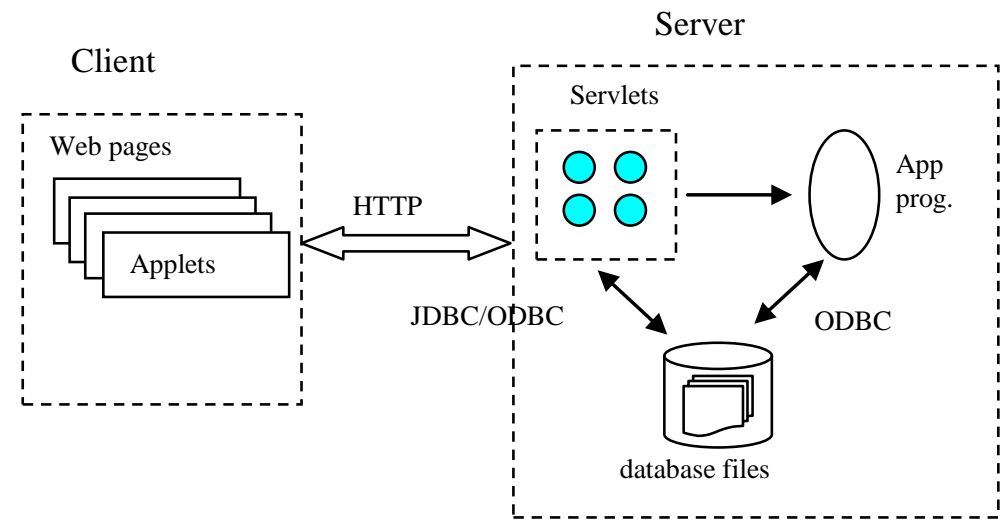

Fig. 1 The system structure

Within the system, the user on the client side can remotely execute, via the Internet explorer in the way of plugging-in, the application program located at the Server side. 
The user interface is written in HTML. The user can input data, monitor the process of the execution, and retrieve resultant data through the interface. During the execution, the application program reads input data from and writes resultant data to database files through ODBC. The Server is a combination of Mirosoft's Internet Information Server (IIS) and Tomcat (a Servlet-enabled server), where the advantages of both types of servers are best utilised while their limitations are avoided.

\subsection{Configuration of the IIS and Tomcat}

In order to apply Servlets in this system, a Servlet-enabled server must be set up. There are a number of Servlet-enabled servers available nowadays, such as Tomcat, JRun, Java Web Server, etc. Tomcat is a worldwide-used Web server built on the Java platform for JSP/Servlets, so it is selected as the Servlets engine in this system.

Compared to Tomcat, Microsoft's Internet Information Server (IIS), which runs on the Windows Server family, has following merits: faster delivery of Web contents and better setting up of SSL (Secure Sockets Layer), giving better control over processor usage and bandwidth usage for Web site, and easier to be operated and maintained in a Windows environment.

Even though IIS has better performance than Tomcat in far more aspects than those listed above, it has a fatal limitation that it is not a Servlet-enabled Web server. To implement Servlets in the system, a good solution is the integration of both IIS and Tomcat. Because no applications can start a server on the same port, IIS and Tomcat must operate on different ports. By default, IIS uses port 80 , which is the default HTTP port, so port number 80 is unnecessary in the URL. Tomcat runs on port 8080 , which means that whenever a Servlet application is referred, port 8080 has to be in the URL. This is not a good software design because it involves some hard coding of addresses and any change leads to modification in source files. When multiple portals and applications run on the server and to integrate all of them in one Web site, hard coding becomes a big issue.

To overcome the hard coding problems, a better solution is to configure IIS and Tomcat working together by utilising the JspISAPI as the bridge between them. All Web requests are sent to IIS through default HTTP port 80. If there is a request for Servlet or a JSP application, then IIS uses the JspISAPI filter to redirect the Servlet or JSP requests to Tomcat. In this way, for those Servlet or JSP requests, no hard coding of port number is needed. Tomcat serves only for Servlet and JSP requests, while the IIS server is responsible for all other requests. The configuration of IIS and Tomcat is illustrated in Fig.2.

Benefits of this configuration are listed below.

- It is not needed to specify 8080 in Web request URL, which helps to improve the software design.

- IIS delivers static contents fast, thus saving processor usage for other Web sites and processes.

- The bandwidth and process of Web sites is controllable.

- It makes Tomcat transparent to the user, even if the port 8080 for Tomcat is blocked by firewall. 
- Configuration of SSL can be easily done on IIS. There is no need to do any changes on Tomcat.

- IIS, by default, offers HTTP Keep Alives, improving the performance.

- IIS also offers HTTP Compression and various modes of caching and content expiration.

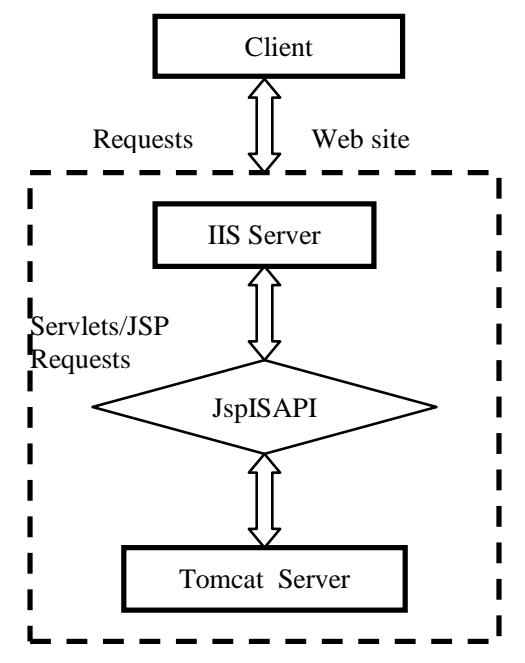

Fig. 2 Configuring IIS and Tomcat Server

\subsection{Implementation of Databases}

Database structure. The system developed has been successfully applied for gear design optimisation [11], where the application program is of genetic algorithms written in $\mathrm{C}++$. Figure 3 shows the structure of a database, called gear_spurhelixd, used for the application program of gear design optimization. Three tables are included in the database, two of which are used for input data, and the other is for resultant data. All the three tables are logically linked by an ID field used to record user's ID number, which is the primary key in the tables.

JDBC-ODBC bridge. ODBC is an API defined by Microsoft. Prior to Sun's development of the Java JDBC API, ODBC was the most widely used programming interface for accessing relational databases. It offers the ability to connect with virtually all databases and platforms. But ODBC uses a $\mathrm{C}$ interface, which is not directly in the Java servlet program. A literal translation of the ODBC C-based API into a Java API would not be feasible due to the extensive use of pointers. ODBC can be used from within the Java platform, which is best done with the help of the JDBC API in the form of the JDBC-ODBC Bridge. The JDBC-ODBC Bridge is itself a JDBC driver defined in the class sun.jdbc.odbc.JdbcOdbcDriver. The Bridge defines the JDBC sub-protocol ODBC. In this application, it is used to connect with Microsoft Access. 
Data flow. In this configuration, the user machine is the client, and the machine hosting the data source is the server. The network can be an intranet or the Internet. In the three-tier model shown in Fig. 1, commands are sent to the "middle tier" of services, e.g. serlvet tier, which then sends the commands to the data source. The data source processes the commands and sends the results back to the middle tier, which then sends them to the user. The three-tier model offers greater control over the kinds of updates that can be made, and it simplifies the deployment of applications.

Setting up Microsoft Access under Windows. The server machine has already been installed with Microsoft Access, so it needs to follow a few setup steps to communicate through the JDBC-ODBC bridge, and then connection to the gear design database can be established.

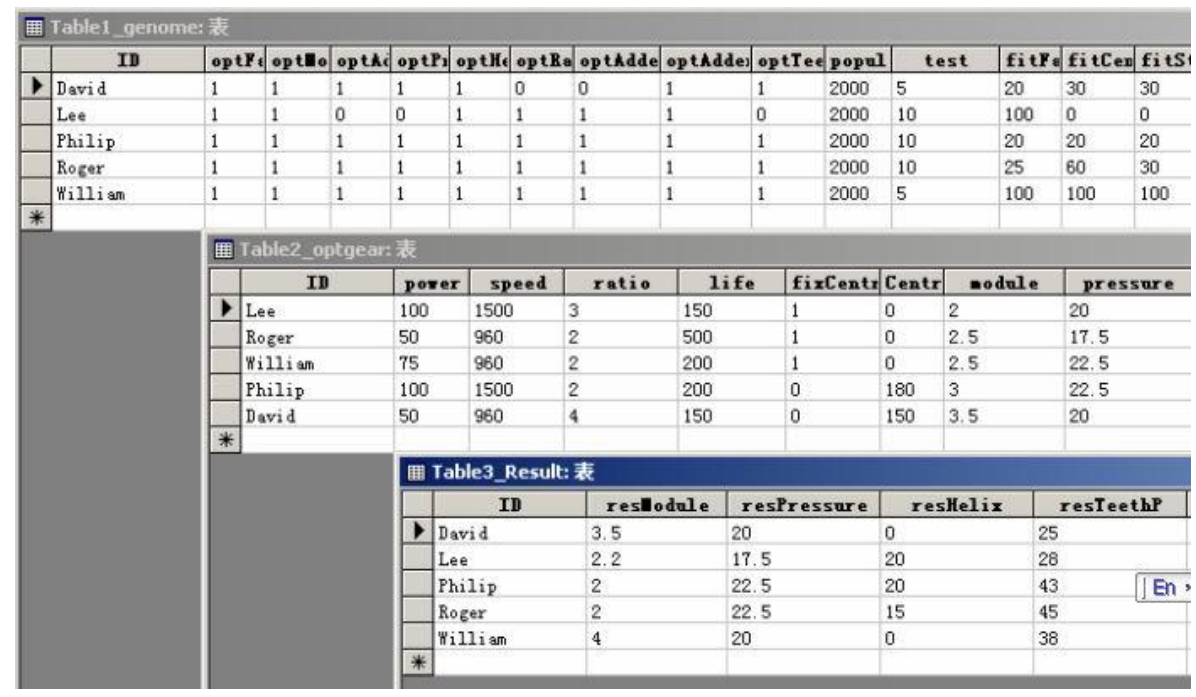

Fig. 3 Structure of a gear design optimisation database

\section{Web-Service Supported Online Collaborative CAD}

\subsection{Main structure of the approach}

In this system, the technology of Web Services plays a key role of connecting an individual site, where the CAD package is located and related services are available to the Internet, with multimedia functions of audio/video and message board. In this research, the popular CAD package, AutoCAD is utilized. The JNI (JAVA Native Interface) carries out the tasks of dynamic data processing and links with the AutoCAD and Web Services. The designer operates the AutoCAD to produce drawings and makes changes which are instantaneously sent, with the aid of JNI and Web Service, to the collaborative designers located in different sites. With the 
multimedia functions, the designers can communicate online in the ways of speaking, writing messages on the board, and seeing each other on the screen during the collaborative design process.

As shown in Figure 4, in order to load a CAD model as a Web service, the provider of the CAD model generates a describing file in WSDL (Web Service Description Language) format first and then registers the service with the WSDL file to the service registration server.

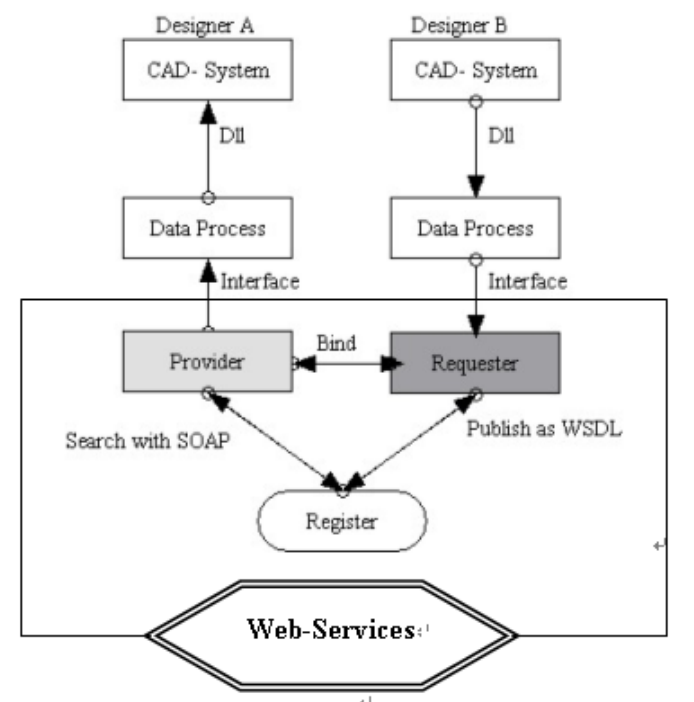

Fig. 4 Operation procedure of the Web supported online CAD

The WSDL file contains all the information of the service including the location of the service. In a normal situation, when a client requests a service, the client, i.e., the requester of the service may do not know where the service is, so he/she searches the registry server, if it is found, the registry server sends the requester the WSDL file. With the information provided in the WSDL file, the client (requester) can find the service. Then the service provider, which is in charge of service allocation, would produce an instance of the service for the request; also, the provider can produce more instances if there are more requests. As shown in Figure 2, when designer B make a change in his AutoCAD drawing, this change would activate outer DLL (Dynamic Linked Library) programme and then invoke a Java interface, which would describe the request as SOAP message. After that, the message would be transferred to designer A who has provided the service, after that, the SOAP message would be analyzed by designer A's system and the AutoCAD would do what is requested, to do the same change in its panel.

\subsection{System Modules}

The system consists of three modules: request module, middleware module and response module 
Request module. This module enables the clients to find and to get the web service via Internet. Taking gear design as an example, the fist step for the gear designer is to draw a circle in the AutoCAD in his local computer; after this is completed, the drawing is sent as a request to another computer (the Server), the system then calls the Java function outside AutoCAD via the $\mathrm{C}++$ program to complete the request.

Middleware module. This module provides functions for the provider to register their service, which is not to operate the service, but provides the service's location and the detail information including methods provided by the service.

In this module, the service providers register their services to the service registry so that the information about the services can be found by requesters via the Internet. Then the service registry directs the service requester to find the right location of the service. After that, the service requester and service provider are bind to each other and ready to correspond each other.

A Java program has been developed containing several parameters including the location of the service registry. The parameters captured by the program are packed in XML format first and then transferred to SOAP message so that the service provider can receive and analyze it.

Response module. This module is located in the Server. It provides functions for the Service provider to response and to react when the requester asks for the web service. When the parameters have been captured from the service, they would be used by the ActiveX automation and if all the procedure goes successfully, the response module would return a signal to inform that the mission is completed.

Note that when the connection is established between two computers, each side can act as either a client or a server. For the case shown in Figure 4, in one action, when designer $\mathrm{A}$ makes a change in the $\mathrm{CAD}$ drawing, the system sends request including the change information to computer $\mathrm{B}$, then computer $\mathrm{B}$ is considered as a server and computer $\mathrm{A}$ is a client in this process, after that when designer $\mathrm{B}$ makes a change, the request module is invoked and designer $\mathrm{B}$ is considered to be a client, and computer A becomes the server.

\subsection{A Case Study}

A case study has been conducted to illustrate the system developed. It includes three computers with PIV $2 \mathrm{G}$ processors located at three sites and linked to the Internet. Each of them is installed with AutoCAD version 2004, Axis, Tomcat 5.0, and Object ARX package. Each computer is also equipped with video and audio facilities including a video camera and a microphone.

The task of the collaborative CAD conducted in this case study is to modify a shaft design online amongst three geographical dispersed designers. When the designers get onto the system, they can see each other from the right side panel on their own computers (Figure 5) and can speak online as well. Each of them can modify the drawing online and any change of the drawing made is simultaneously displayed on the three computers, for further details see [12]. 


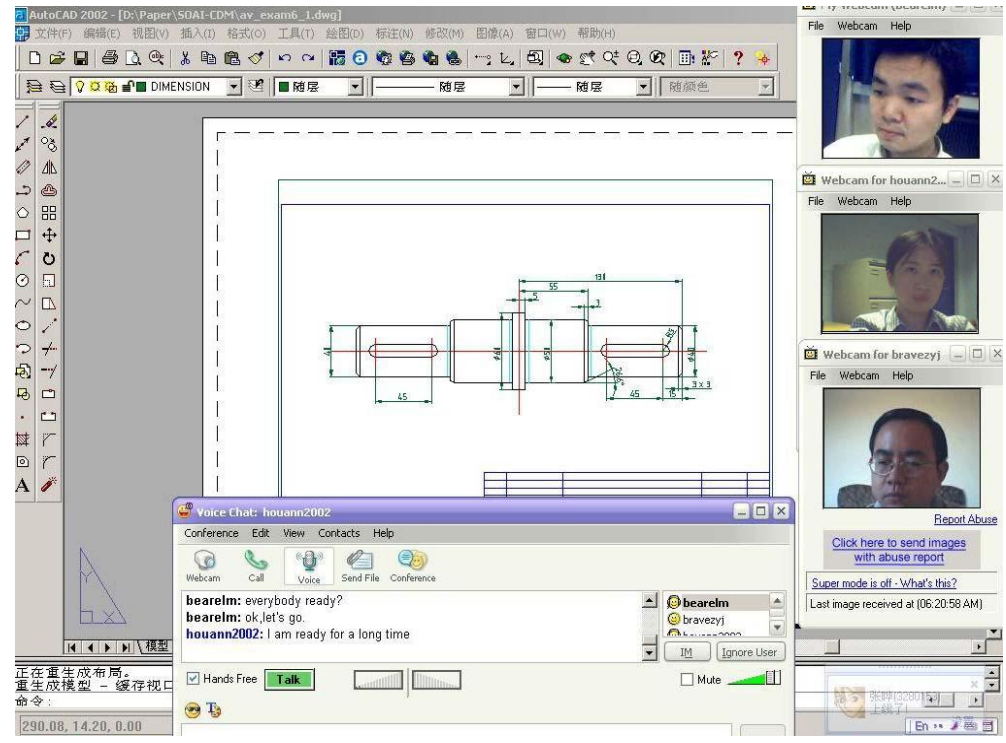

Fig. 5 An example of online collaborative CAD

\section{Concluding Remarks}

The research in Web-enable collaborative design and manufacture has been conducted and reported in this paper. The major tasks, where the Web/Internet techniques are heavily involved, include a Web-enable environment for collaboration, online collaborative CAD/CAM, remote execution of large size programs, and distributed product design.

The client-server approach developed in this research provides an important solution for remote execution of large-size programs without downloading the program. The combination of IIS and Tomcat provides a powerful combined server, which utilizes the advantages of the two types Servers. The integration of JDBC and ODBC makes the databases work in an effective way.

The Web-Services supported collaborative CAD enables geographically dispersed designers jointly conduct a design task in the way of speaking and seeing each other and instantaneously modifying the CAD drawing online. Currently AutoCAD has been utilised in the system. As a further development, deferent CAD packages such as ProEngineer and Solid Works will be considered, so that online collaboration across different CAD environments can be achieved.

\section{Acknowledgement}

The authors are grateful for the support received from the EU Asia-Link programme (grant No.ASI/B7-301/98/679-023) and Asia IT\&C programme (Grant No. ASI/B7- 
301/3152-099/71553) for carrying out the research. The authors acknowledge contributions from team members of the two project consortia including Labein Technology Centre (Spain), Harbin Institute of Technology (China), Chongqing University (China), Lappeerata University of Technology (Finland) and Nottingham Trent University (UK).

\section{References}

1. F.T.S. Chan, J. Zhang, P. Li, 2003, "Agent- and CORBA-based application integration platform for an agile manufacturing environment", International Journal of Advanced Manufacturing Technology, 21(6), 2003, pp 460-468

2. Marek B. Zaremba and Gerard Morel, 2003, "Integration and control of intelligence in distributed manufacturing", Journal of Intelligent Manufacturing, 14 (1), 2003, pp. 25-42.

3. J C Hou, D Su and J B Hull, 2004, 'Integration of Web-based Techniques and Business Strategies into the Development of a Network Supported System for Supplier Selection', $8^{\text {th }}$ International Conference on Computer Supported Cooperative Work in Design, 26-28 May 2004, Xiamen, China, pp 599-604

4. EU Asia IT\&C project, 'Web-enabled collaboration in intelligent design and manufacture', Contract No. ASI/B7-301/3152-99/72553.

5. EU Asia-Link project, 'Nottingham Trent-Lappeenranta-Chonqing Universies' collaboration for human resource development in mechanical and manufacturing engineering', Contract No. ASI/B7-301/98/679-023

6. Jiansheng Li, Daizhong Su, J L Henshall and Yu Xiong, 'Development of a Web-enabled Environment for Collaborative Design and Manufacture', proceedings, $8^{\text {th }}$ International Conference on Computer Supported Cooperative Work in Design, 26-28 May 2004, Xiamen, China, pp 540-545.

7. Xiaoan Chen, Tianhong Luo, Ye He, Wei Zhou and Dongmei Sun, 'Research on 3D Feature Modeling technology for Internet-driven Collaborative Design', $9^{\text {th }}$ International Conference on Computer Supported Cooperative Work in Design, 24-26 May 2005, Coventry, UK, pp 649-654 .

8. Shisheng Zhong, Yan Zhang, Lin Lin and Daizhong Su, 'Development of a Web-based Collaborative Manufacturing System for Parallel Kinematic Machines', $9^{\text {th }}$ International Conference on Computer Supported Cooperative Work in Design, 24-26 May 2005, Coventry, UK, pp 667-672.

9. D Su, and N Amin, 2001, 'A CGI-based approach for remotely executing a large program for integration of design and manufacturing over the Internet', International Journal of Computer Integrated Manufacturing, Vol 14, No.1, pp 55-65.

10. Inigo Mendikoa, Mikel Sorli, Jose Barbero and Ana Carrillo, 'Knowledge based distributed product design and manufacturing', $9^{\text {th }}$ International Conference on Computer Supported Cooperative Work in Design, 24-26 May 2005, Coventry, UK, pp 679-684.

11. Shuyan Ji, Daizhong Su, J L Henshall and J B Hull, 2004, 'Gear Design Optimisation Using A Genetic Simulated Annealing Algorithm', poster proceedings, International Conference on Adaptive Computing in Design and Manufacture, 20 - 22 April 2004, Bristol, UK, pp 5-9.

12. Yu Xiong, 2005, 'Application of Advanced Web/Internet techniques into the development of the Virtual Research Institute', Report for transfer from MPhil to PhD degree studies, Nottingham Trent University, May 2005. 